\title{
Brillouin-Based Distributed Temperature Sensor Employing Pulse Coding
}

\author{
Marcelo A. Soto, Prasant K. Sahu, Gabriele Bolognini, and Fabrizio Di Pasquale, Member, IEEE
}

\begin{abstract}
A distributed temperature sensor based on spontaneous Brillouin scattering and employing optical pulse coding has been implemented and characterized using a direct-detection receiver. The signal-to-noise ratio (SNR) enhancement provided by coding is analyzed, along with the influence of coding in stimulated Brillouin threshold. Simplex-coding using 127 bit codeword provides up to $7 \mathrm{~dB}$ SNR improvement, allowing for temperature sensing over $21 \mathrm{~km}$ of dispersion shifted fiber with $3.1 \mathrm{~K}$ resolution and $40 \mathrm{~m}$ spatial resolution, permitting to avoid the use of optical pulse amplification.
\end{abstract}

Index Terms-Brillouin scattering, distributed-fiber sensor, optical time-domain reflectometry (OTDR).

\section{INTRODUCTION}

Distributed temperature sensors employing optical fiber as the sensing element exploit most commonly nonlinear effects such as Raman and Brillouin scattering.

Compared with the well-known Raman-based distributed temperature sensors (DTS) [1], Brillouin-based DTS (BDTS) are characterized by higher backscattered intensities, are less sensitive to wavelength dependent losses, but generally need more complex receiver schemes; in addition, they are sensitive to strain as well, allowing for the possibility to implement combined temperature and strain sensing [2]. BDTS can be implemented exploiting either spontaneous Brillouin scattering (SpBS) or stimulated Brillouin scattering (SBS) effects together with OTDR [3]; while SpBS requires a simpler implementation schemes, SBS allows for higher signal-to-noise ratio (SNR) levels and higher accuracy, but requires access to both fiber ends and a more complex receiver scheme.

In this letter, we have characterized the enhanced performance of SpBS-based DTS employing optical pulse coding (namely, Simplex coding) for the first time to our knowledge. We experimentally show that Simplex coding can greatly improve the receiver SNR, allowing for more accurate temperature sensing, and that coding is characterized by lower optimum input peak power (before SBS onset) compared with single-pulse operation avoiding the use of optical pulse amplification.

\section{THEORY}

In order to investigate the characteristics of pulse coding in SpBS-based sensors, we have implemented an OTDR-based distributed temperature sensor using the Landau-Placzek ratio (LPR) scheme [3], and applied Simplex-coding for SNR-en-

Manuscript received August 2, 2007; revised October 4, 2007; accepted October 5, 2007. The associate editor coordinating the review of this paper and approving it for publication was Prof. Francisco Arregui.

M. A. Soto, G. Bolognini, and F. Di Pasquale are with Scuola Superiore Sant'Anna, Pisa 56124, Italy (e-mail: gabriele.bolognini@sssup.it).

P. K. Sahu is with the Indian Institute of Technology, Kharagpur 721302, India.

Digital Object Identifier 10.1109/JSEN.2007.913143

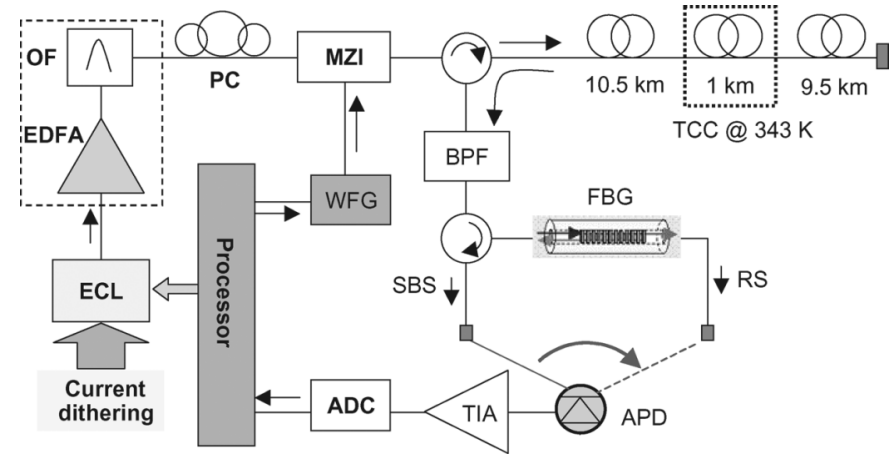

Fig. 1. Experimental setup of implemented BDTS.

hancement in direct-detection of SpBS. The temperature dependence of LPR is given by the well-known formula

$$
\frac{I_{R S}}{I_{S p B S}}=\frac{T_{f}}{T}\left(\beta_{T} \rho_{0} v_{s}^{2}-1\right)
$$

where $\beta_{T}$ is the isothermal compressibility, $\rho_{0}$ the density, $T_{f}$ the fictive temperature, $v_{s}$ the acoustic velocity, and $I_{\mathrm{RS}, \mathrm{SpBS}}$ are the intensities of Rayleigh scattering (RS) and SpBS. If no optical nonlinearities occur along the fiber, the temperature resolution is mainly dictated by the noise level at the receiver.

The use of pulse coding in this case can, thus, provide a considerable SNR enhancement. Benefits of coding can be quantified by the coding gain, defined as the ratio of the SNR obtained with coded pulses to the SNR obtained with single pulses at a given distance, and equal number of total measured traces. For Simplex coding, the coding gain $G_{\mathrm{COD}}$ for an arbitrary code length $L$ is given by [4]: $G_{\mathrm{COD}}=(L+1) /(2 \sqrt{ } L)$.

The successful use of Simplex coding in Raman-based DTS has been already demonstrated in [5]; however, the effectiveness of coding with Brillouin-based sensors is not straightforward since the physical effects underlying Brillouin DTS are substantially different from Raman-DTS. One important difference is the narrower bandwidth of Brillouin gain, making the use of narrowband sources necessary for Brillouin sensors (compared with broadband sources used in Raman sensors). This feature can have a strong impact on effectiveness of coding techniques in SpBS-based sensors, since coded pulses can greatly affect the threshold for onset of SBS, while coding used for Raman-based sensors have negligible impact on the threshold for stimulated Raman scattering. In this letter, we show that SpBS-based DTS systems using coded OTDR are characterized by low optimum input peak power levels before SBS onset, avoiding the use of costly optical amplifiers, and that coding is able to provide more accurate temperature measurements over long distances.

\section{EXPERIMENTAL SETUP}

The setup used to study BDTS employing pulse coding is shown in Fig. 1; coded OTDR technique is used with a direct- 

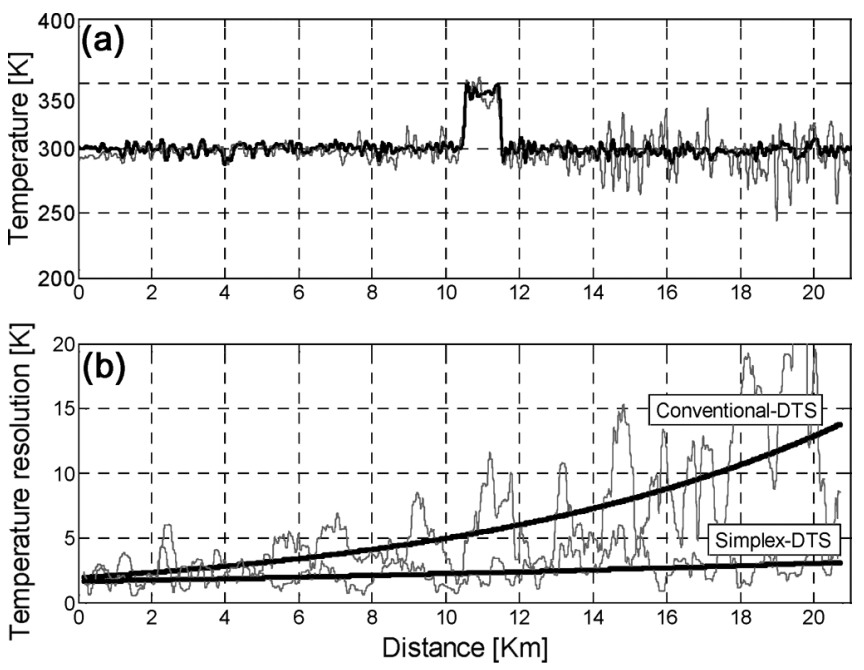

Fig. 2. (a) Temperature distribution along the fiber using single pulses (gray line) and Simplex-coded pulses (black line), with mid-spool heated at $340 \mathrm{~K}$ (b) Achieved temperature resolution (fitting the rms of temperature distribution).

detection receiver block. The used source is an external cavity laser (ECL) tuned at $1550.1 \mathrm{~nm}$ (200 kHz linewidth), which is amplified by an EDFA, optically filtered through a tunable bandpass filter (OF, $0.8 \mathrm{~nm}$ FWHM) to reduce ASE noise, polarizationcontrolled and then modulated by a Mach-Zehnder modulator (MZI) to provide single pulses, as well as 127-bit Simplex-coded pulses (400 ns pulsewidth, resulting in $40 \mathrm{~m}$ resolution) through a waveform generator (WFG). For achieving high peak power values in single-pulse BDTS, the EDFA, and OF can be moved after the MZI (not shown in Fig. 1). The coded light pulses are then coupled through an optical circulator into the sensing fiber which is composed of three spools of dispersion-shifted fiber (DSF), with 10.5, 1, and $9.5 \mathrm{~km}$ length, respectively. The temperature of the mid-DSF spool has been raised during experiments by placing it inside a temperature-controlled chamber (TCC), while the remaining spools have been kept at room temperature $(300 \mathrm{~K})$.

Current dithering has been applied to the laser source in order to broaden its linewidth, hence increasing the power threshold value before the onset of detrimental SBS, and also helping to mitigate coherent Rayleigh noise (CRN). Also, $\lambda$-averaging over $60 \mathrm{GHz}$ was used in Rayleigh scattering measurements to reduce CRN down to $0.37 \%$. The receiver block detecting the backscattered light from the sensing fiber is based on a low-loss direct-detection scheme, composed of an optical circulator combined to a narrowband fiber Bragg grating (FBG, $6 \mathrm{GHz}$ bandwidth), separating the anti-Stokes Brillouin-scattered light from the Rayleigh-scattered light ( $28 \mathrm{~dB}$ band rejection) into two different circulator ports. The detector is given by an avalanche photodiode (APD), followed by a high-gain transimpedance amplifier (TIA) and an analog-digital converter (ADC) stage.

\section{RESULTS}

The temperature estimation along the sensing fiber has been derived from LPR [3]. In order to have a reliable comparison, results were obtained for the same measurement time for both single-pulse and Simplex-coded DTS (180 K total acquired

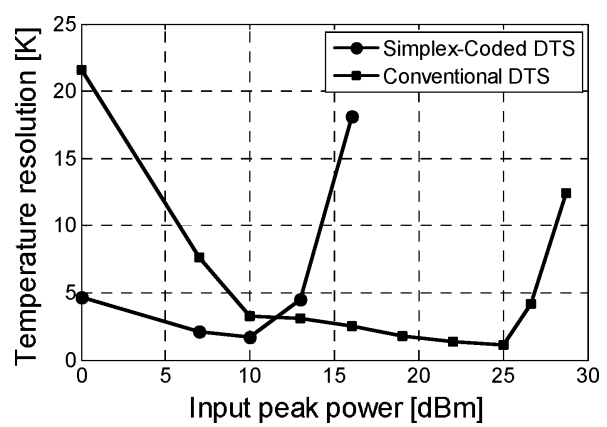

Fig. 3. Temperature resolution (at $4 \mathrm{~km}$ distance) versus input peak power using single pulses (squares) and Simplex-coded pulses (circles).

traces in both cases, allowing for an attainable acquisition time lower than 1 min excluding processing overhead). Fig. 2(a) compares the temperature distributions when the TCC was set to $343 \mathrm{~K}$ (input peak power $10 \mathrm{~mW}$ ). It is evident that the temperature accuracy with coded-BDTS is better than with single-pulse BDTS, thanks to the SNR enhancement using Simplex (measured $G_{\mathrm{COD}}=7.12 \mathrm{~dB}$ in agreement with theoretical value $G_{\mathrm{COD}}=7.54 \mathrm{~dB}$ ). The improved temperature resolution (fitting the rms of the difference between estimated and real temperature) is shown in Fig. 2(b), and is equal to $3.1 \mathrm{~K}$ at $21 \mathrm{~km}$ with coded-BDTS (instead of $13.7 \mathrm{~K}$ with single pulses).

Fig. 3 shows the temperature resolution versus input peak power, for both Simplex-coded and single-pulse BDTS at $\mathrm{L}=4 \mathrm{~km}$ (near fiber input). Similar optimal resolution values are found for both cases, but, since the coding process results in a longer pulse effective length (thus decreasing the SBS threshold), the optimal resolution with Simplex-BDTS is found at lower input peak power values than with single-pulse BDTS. This allows accurate temperature sensing at low peak power levels (about $15 \mathrm{~dB}$ lower than for single pulse), avoiding the use of optical pulse amplification.

In conclusion, we have shown experimentally the advantage of Simplex coding technique in BDTS using LPR for temperature resolution enhancement. Results show that Simplex-coding allows a temperature resolution similar to a single-pulse case at much lower input peak power. Coding techniques can then possibly be applied as an alternative to optical amplification in BDTS, providing a high-performance cost-effective solution.

\section{REFERENCES}

[1] M. A. Farahani and T. Gogolla, "Spontaneous Raman scattering in optical fibers with modulated probe light for distributed temperature Raman remote sensing," J. Lightw. Technol., vol. 17, pp. 1379-1391, 1999.

[2] R. Bernini, A. Minardo, and L. Zeni, "A reconstruction technique for stimulated Brillouin scattering fiber-optic sensors for simultaneous measurement of temperature and strain," in Proc. IEEE Sensors, 2002, pp. 1006-1011.

[3] K. De Souza and T. P. Newson, "Improvement of signal-to-noise capabilities of a distributed temperature sensor using optical preamplification," Meas. Sci. Technol., vol. 12, pp. 952-957, 2001.

[4] D. Lee et al., "Analysis and experimental demonstration of simplex coding technique for SNR enhancement of OTDR," in Proc. IEEE LTIMC, New York, Oct. 2004, pp. 118-122.

[5] J. Park et al., "Raman-based distributed temperature sensor with Simplex coding and link optimization," IEEE Photon. Technol. Lett., vol. 18, pp. 1879-1881, 2006. 